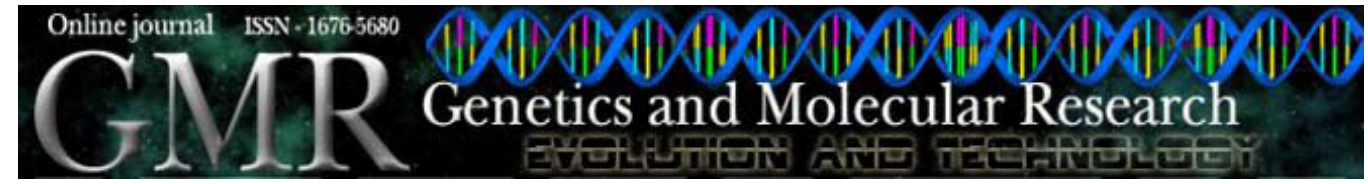

\title{
Mediterranean fever gene mutation analysis in infertile Turkish males
}

\author{
E.O. Etem ${ }^{1}$, D. Erol ${ }^{1}$, Y. Huseyin ${ }^{2}$ and A. Arslan ${ }^{2}$ \\ ${ }^{1}$ Department of Medical Biology and Genetics, \\ Faculty of Medicine, Firat University, Elazig, Turkey \\ ${ }^{2}$ Department of Urology, Faculty of Medicine, \\ Firat University, Elazig, Turkey \\ Corresponding author: E.O. Etem \\ Email: ebruetem@gmail.com
}

Genet. Mol. Res. 9 (2): 611-619 (2010)

Received December 20, 2009

Accepted January 15, 2010

Published April 6, 2010

DOI 10.4238/vol9-2gmr743

\begin{abstract}
Male infertility is a common barrier that prevents successful conception. There have been reports of azoospermia in men with familial Mediterranean fever, some of whom had not been treated with colchicine. Variation in this disorder could be a risk factor for amyloidosis associated with azoospermia. We determined the frequency of 6 of the most common Mediterranean fever gene mutations, M680I, M694V, M694I, V726A, P369S, and A744S, in 74 infertile men, 155 men diagnosed with familial Mediterranean fever and 55 healthy fertile men in eastern Turkey. All three groups were screened for the 6 mutations using an amplification refractory mutation system and restriction fragment length polymorphism methods. Allelic frequencies were $2.7 \%$ for M694V and $1.35 \%$ for V726A in the infertile patient group and $1.8 \%$ for M694V and $1.8 \%$ for V726A in healthy subjects. Other mutations were not detected in patients or controls. The mutation frequency was not found to be significantly higher in infertile patients when compared with healthy fertile male controls. To our knowledge, this is the first study to determine the frequency of Mediterranean fever gene mutations in infertile male and the infertility rate of male patients with familial Mediterranean fever.
\end{abstract}

Key words: Familial Mediterranean fever; Male infertility; Mediterranean fever gene 


\section{INTRODUCTION}

Infertility is frequently attributed to defects of sperm motility and number. The current estimate is that about $30 \%$ of men seeking help at the infertility clinic are found to have oligospermia or azoospermia of unknown etiology. Numerous factors contribute to male infertility, including chromosomal aberrations, gene defects, hormonal milieu, genital infections, and chemical and physical agents. The examination of male infertility should be complex, including detailed history, physical examination, semen analysis, hormonal screening, and chromosomal and genetic analysis of somatic cells (Van Assche et al., 1996).

Familial Mediterranean fever (FMF) is the most frequent periodic syndrome characterized by recurrent attacks of polyserositis. The disease affects mainly Jews, Armenians, Arabs, and Turks (Eliakim et al., 1981). Very high FMF carrier rates have been described among the Mediterranean and Middle Eastern populations, ranging from 1:5 among North African Jews, Arabs, and Turks to 1:3 among Iraqi Jews and Armenians. Mediterranean fever (MEFV) gene maps to chromosome $16 \mathrm{p}$, and encodes a 781-amino acid protein, called pyrin or marenostrin, which acts as a repressor of a proinflammatory molecule or a transcriptional up-regulator of an anti-inflammatory protein (Sayarlıoglu et al., 2005). The most severe manifestation of FMF results from the deposition of amyloid A protein. This protein is presumed to be a cleavage product of serum amyloid A, an acute-phase reactant produced by the liver. Amyloidosis may lead to male and female infertility through amyloid deposition in testes and ovaries (Ben-Chetrit et al., 1998).

Our knowledge about the effect of FMF on male fertility is quite limited. In patients with FMF with azoospermia, colchicine was considered to be the sole and direct cause. Controversial results have been published on the effect of colchicine on spermatozoa in humans (Merlin, 1972; Gonzalez et al., 1983; Kastrop et al., 1999; Haimov-Kochman et al., 2001). Sporadic reports described that colchicine affects sperm production and function (Gonzalez et al., 1983; Kastrop et al., 1999). It is assumed, though, that some men are unusually sensitive to the toxic effect of colchicine on the testis, which probably could explain the rare cases of azoospermia observed with colchicine treatment (Merlin, 1972). Weighing the available evidence, our impression is that the frequency of colchicine-induced sperm pathology in FMF patients is relatively low and that the occurrence of oligospermia or azoospermia may depend primarily on testicular pathology related to FMF itself (Ben-Chetrit et al., 1998). This was based on a case report from the early 1970's, in which Merlin (1972) described azoospermia in a patient with gout after chronic colchicine treatment. However, during the past few years, several men with FMF and azoospermia have been encountered, some of whom did not receive colchicine (BenChetrit et al., 1998; Haimov-Kochman et al., 2001).

We detected two infertile cases in 155 male patients with FMF. Therefore, we decided to study the common MEFV gene mutations in infertile Turkish azoospermia or oligoospermia males compared with a healthy fertile male group. The main purpose of this study was to investigate the possible MEFV mutation frequency of infertile Turkish men compared with healthy fertile male controls, to determine the rate of infertility in FMF diagnosed patients and to detect whether a specific mutation is the cause of infertility in FMF or infertile male patients. 


\section{MATERIAL AND METHODS}

The Ethics Committee of the Faculty of Medicine at Firat University approved this study. We explained the purpose and scope of the study to all eligible subjects and invited them to participate. For participation in the study, all patients provided written informed consent.

\section{Patients}

Three different groups were included in this study. The first group comprised 74 patients who were diagnosed clinically between 2006 and 2008 as having oligospermia and azoospermia. The patients in the second group had been clinically and genetically diagnosed with FMF $(\mathrm{N}=155)$. This group was analyzed retrospectively according to the records of the patients referred to the Department of Medical Biology and Genetics at Firat University from January 2004 to August 2009. The third group consisted of control individuals $(\mathrm{N}=55)$ who were genetically similar to the infertile male group and pregnancy-proven fertile male controls. The three different groups lived in the East Anatolia region of Turkey.

Couples who failed to conceive after 1 year of unprotected sexual intercourse were defined as being infertile. The male partners in such couples, who attended the Infertility Clinic at Firat University Hospital, were recruited into the study. They were asked to complete a comprehensive questionnaire regarding their medical and surgical history (such as cryptorchidism in childhood, genital infections, trauma, and operations), lifestyle habits (such as smoking, alcohol and drug use), and exposure to gonadotoxins (such as radiation therapy and drugs used for cancer chemotherapy). Examination for the presence of varicocele was carried out by clinical examination only.

A complete semen analysis was performed in all cases according to the World Health Organization Guidelines (Rowe et al., 2000). Semen samples were obtained after a 2- to 7-day period of sexual abstinence. The patients were either azoospermic, having no sperm in the ejaculate $(\mathrm{N}=46)$ or severely oligozoospermic, having a sperm count of less than 5 million $/ \mathrm{mL}(\mathrm{N}$ $=28$ ). In addition, blood samples were obtained for DNA extraction and chromosome analysis.

Chromosome analyses were performed in our Genetics Laboratory, Faculty of Medicine, Firat University. Briefly, cultures of peripheral blood lymphocytes were treated with 0.1 $\mu \mathrm{g} / \mathrm{mL}$ colcemid (Sigma, St. Louis, USA) after a 72-h incubation period. The prometaphase chromosomes were spread and stained using standard G- and Q-banding techniques. At least 20 metaphases per subject were analyzed.

\section{Mutation analysis}

Venous blood was drawn from each patient using standard techniques and genomic DNA was isolated from peripheral blood leukocytes using a Promega Wizard DNA Extraction kit (Promega, USA). Mutation identification in infertile male patients and healthy controls was performed according to previously described techniques (Anonymous, 1997; Eisenberg et al., 1998; Medlej-Hashim et al., 2000). The mutations were assessed by amplifying the genomic DNA template with three sets of normal and mutant specific amplification refractory mutation system primers designed to selectively amplify the normal or altered sequence of each of the FMF gene mutations. The sequences of the primers are shown in Table 1. The amplicons were 
amplified in a $20-\mu \mathrm{L}$ reaction mixture consisting of $50 \mathrm{ng}$ genomic DNA, 10 pmoL primer (Iontek, Istanbul, Turkey), $4.0 \mathrm{mM} \mathrm{MgCl}, 0.2 \mathrm{mM}$ dNTP and $0.5 \mathrm{U}$ of Taq DNA polymerase. All PCR procedures were carried out as follows: initial denaturation at $95^{\circ} \mathrm{C}$ for $5 \mathrm{~min}$, followed by 35 cycles of denaturation at $95^{\circ} \mathrm{C}$ for $30 \mathrm{~s}$, annealing at $56^{\circ} \mathrm{C}$ for $30 \mathrm{~s}$ and extension at $72^{\circ} \mathrm{C}$ for $30 \mathrm{~s}$, with a final elongation at $72^{\circ} \mathrm{C}$ for $5 \mathrm{~min}$. PCR products were analyzed on a $2 \%$ agarose gel and photographed on a UV light transilluminator. Diagnosis of male FMF patients was established according to previously described criteria (Yalcinkaya et al., 2000). Patients had been referred from a number of medical centers in the East Anatolia region of Turkey. All of them were interviewed directly by one of the clinicians with identical clinical forms. Mutation analysis in male FMF patients was performed by the StripAssay technique (Vienna Lab., FMF StripAssay GmbH, Austria). We use this kit in our Genetics Laboratory for routine MEFV gene mutation screening of FMF diseases.

\begin{tabular}{|c|c|c|}
\hline Mutation & Primer sequences & Restriction enzyme \\
\hline \multirow{3}{*}{ M680I } & C: 5'-TTAGACTTGGAAACAAGTGGGAGAGGCTGC-3' & \\
\hline & M: 5'-ATTATCACCACCCAGTAGCCATTCTCTGGCGACAGAGCG-3' & \\
\hline & N: 5'-ATTATCACCACCCAGTAGCCATTCTCTGGCGACAGAGCC-3' & \\
\hline \multirow{3}{*}{ M694V } & C: 5'-TATCATTGTTCTGGGCTC-3' & \\
\hline & M: 5'-GGTACTCATTTTCCTTCAC-3' & \\
\hline & N: 5'-TGGTACTCATTTTCCTTCAT-3' & \\
\hline \multirow[t]{3}{*}{ M694I } & C: 5'-TATCATTGTTCTGGGCTC-3' & \\
\hline & M: 5'-CTGGTACTCATTTTCCTTT-3' & \\
\hline & N: 5'-CTGGTACTCATTTTCCTTC-3' & \\
\hline \multirow[t]{3}{*}{ V726A } & C: 5'-GGAGGTTGGAGACAAGACAGCATGGATCC-3' & \\
\hline & M: 5'-TGGGATCTGGCTGTCACATTGTAAAAGGAGATGCTTCCTG-3' & \\
\hline & N: 5'-TGGGATCTGGCTGTCACATTGTAAAAGGAGATGCTTCCTA-3' & \\
\hline \multirow{2}{*}{ P369S } & F:5'-GAAGAGCCCGGGAAGCCTGAGC-3' & SacI \\
\hline & R:5'-TTGGGAAAATGAAGTAAGGCCC-3' & \\
\hline \multirow{3}{*}{ A744S } & C:5'-GAGGTGGAGGTTGGAGACAA-3' & \\
\hline & M:5'-CCAGAGAAAGAGCAGCTGGA-3' & \\
\hline & N:5'-CCAGAGAAAGAGCAGCTGGC-3' & \\
\hline
\end{tabular}

\section{Statistical analysis}

Comparisons of patient and control groups were made using the $t$-test. Genotype frequency comparisons were made using the $\chi^{2}$ test. $\mathrm{P} \leq 0.05$ was accepted as statistically significant.

\section{RESULTS}

The mean ages (mean) of infertile males, controls and FMF patients were 31 years (range 24-45), 28 years (range 21-45), and 37 years (range 15-61), respectively. The average duration of infertility in infertile males was 8 years (range 2-26 years). None of the cases reported previous exposure to gonadotoxins, such as radiation treatment or cancer chemotherapy. Traditional karyotyping was performed on control and infertile male groups. The chromosomal analysis from peripheral blood lymphocytes revealed a normal male karyotype in all cases.

In the current study, MEFV gene mutations were detected in $3(4.05 \%)$ of 74 infertile males and in $2(3.63 \%)$ of 55 controls, and no mutations were found in the remaining ca- 
ses. We found the following frequencies of MEFV gene mutation in infertile patients: $2.7 \%$ $(2 / 74)$ for M694V and $1.35 \%(1 / 74)$ for V726A. The frequencies of MEFV gene mutation in controls were 1.8\% (1/55) for M694V and 1.8\% (1/55) for V726A. M680I, M694I, P369S, and $\mathrm{A} 744 \mathrm{~S}$ mutations were not detected in the patient or control group. We revealed that the allele frequency of MEFV mutations was not significantly different between the infertile male patients and fertile male controls $(\mathrm{P}>0.05)$.

The frequency of azoospermia or oligospermia among all FMF patients was found to be $1.2 \%(2 / 155)$. The patient with azoospermia had an M680I/M680I genotype, and the patient with oligospermia had an M694V/M694V genotype. The patient carrying MEFV M680I/M680I mutation also developed amyloidosis (patient's age: 54 years), but amyloidosis was not shown in the oligospermic case (patient's age: 32 years). Forty-five (29.0\%) of the 155 male FMF patients were homozygotes, and 73 (47.0\%) were compound heterozygotes. Thirty-seven $(23.8 \%)$ patients had only a single mutation. The distribution of FMF mutations in the study groups is given in Table 2 .

\begin{tabular}{|c|c|}
\hline Patients with FMF ( $\mathrm{N}=155$ patients $/ 310$ alleles $)$ & Allele frequency $(\%)$ \\
\hline M694V & $71(22.9 \%)$ \\
\hline E148Q & $62(20 \%)$ \\
\hline V726A & $42(13.5 \%)$ \\
\hline M680I & $25(8.0 \%)$ \\
\hline P368S & $12(3.8 \%)$ \\
\hline $\mathrm{R} 761 \mathrm{H}$ & $8(2.5 \%)$ \\
\hline A744S & $6(1.9 \%)$ \\
\hline K695R & $4(1.2 \%)$ \\
\hline M694I & $4(1.2 \%)$ \\
\hline F479L & $2(0.6 \%)$ \\
\hline Other/undefined mutation & $74(23.8 \%)$ \\
\hline Infertile patients $(\mathrm{N}=74)$ & \\
\hline M694V & $2(2.7 \%)$ \\
\hline V726A & $1(1.3 \%)$ \\
\hline Control subjects $(\mathrm{N}=55)$ & \\
\hline M694V & $1(1.8 \%)$ \\
\hline V726A & $1(1.8 \%)$ \\
\hline
\end{tabular}

\section{DISCUSSION}

In the present study, we report the frequencies of the six most common MEFV mutations (M680I, M694V, M694I, V726A, A744S, and P369S) in infertile Turkish male patients compared with healthy fertile control subjects and the infertility rate in untreated FMF male patients. The frequency of the MEFV gene mutation in infertile patients was not significantly increased compared with fertile controls. To the best of our knowledge, this is the first study performed to investigate MEFV gene mutation in an infertile male patient group and the infertility rate in untreated FMF male patients.

It is clear that there are many genetic factors leading to infertility such as cytogenetic abnormalities, microdeletions of chromosome $\mathrm{Y}$, and mutations in the genes responsible for sperm production, including CTFR, SOX9, KALIG1, and AKAP82 (Shah et al., 2003). To create a specific model for the interpretation of male infertility data in FMF disease may be difficult. A rela- 
tionship between FMF diseases and infertility has been reported among severely oligozoospermic and azoospermic men (Ben-Chetrit et al., 1998). Amyloidosis, specific MEFV gene mutations and colchicine treatment may be important causes of infertility in male patients with FMF.

First, amyloidosis is the second major manifestation of FMF. It has been diagnosed in $28 \%$ of those reported in the literature (Sohar et al., 1967). The association between testicular amyloidosis and secondary azoospermia is unclear. Usually, FMF-associated amyloidosis of the AA type involves the kidneys, liver, spleen, heart, and intestines (Fonnesu et al., 2009). Testicular amyloidosis is a rare complication of FMF, which is usually encountered in colchicine-resistant or non-compliant patients with clinical signs of renal amyloidosis. Biopsy samples from FMF infertile azoospermia male testes revealed marked germ cell aplasia or maturation arrest of the spermatocytes with amyloid deposition in the blood vessels. It is still unknown whether amyloid disturbs sperm transport by obliteration of intratesticular canaliculi, causing obstructive azoospermia, or disrupts sperm production by its direct effect on the seminiferous tubules. Recently, it has been reported that the development of amyloidosis in Behçet diseases, which may also affect the testicular blood vessels as well as seminiferous tubules, may mimic the vasculitic pathology of Behçet disease in predisposing to azoospermia (Sarica et al., 1995; Haimov-Kochman et al., 2001). Amyloidosis of the testicular blood vessels in azoospermic or oligospermic FMF patients may have a role parallel to vasculitis in Behçet's disease in predisposing to azoospermia while on colchicine therapy. Amyloidosis and the mean age of onset of disease may be two important reasons for infertility in our azoospermic case. Because delayed diagnosis in the patients with advanced age can increase the likelihood of amyloidosis development, early diagnosis and colchicine treatment may be two important criteria in the prevention of the amyloidosis and the development of infertility in male FMF patients.

Secondly, colchicine is effective in suppressing episodes in more than $90 \%$ of patients and prevents both the development of amyloidosis and the additional deterioration of renal function in those with early amyloidosis (Ben-Chetrit and Levy, 1991). Animal data showing a toxic effect of colchicine at 30-50 times higher than the usual dose (1-2 mg per day) on sperm production resulting in spermatogenic arrest and azoospermia have been reported (Handel, 1979; Liang et al., 1985). Therefore, it was not surprising when normal semen analyses and hormonal profiles were found in healthy volunteers (Bremmer and Paulsen, 1976) as well as in FMF patients (Levy and Yaffe, 1978) on continuous colchicine. Ben-Cherit et al. (1998) detected $2(1.3 \%)$ cases with azoospermia or oligospermia in 150 male FMF patients treated with colchicine. It should be emphasized that these patients did not have amyloidosis or renal failure. Another report of 19 male FMF patients receiving colchicine prophylaxis revealed fertility disturbances in 4 of them (21\%) while on treatment (Ehrenfeld et al., 1986). This observation suggests that the risk of colchicine-induced azoospermia in FMF patients with amyloidosis and/or with uremia is higher compared to those with FMF only. Weighing the available evidence, our impression is that the frequency of colchicine-induced sperm pathology in FMF patients is relatively low and that the occurrence of oligospermia or azoospermia may be dependent on testicular pathology related to FMF itself, because infertility rates of FMF male patients treated or non-treated with colchicine were similar.

Although genotype-phenotype correlations and infertility rates of colchicine-treated patients in most of studies related to FMF have been discussed in detail, there are no data in the literature regarding infertility rate in patients not treated with colchicine (Shinar et al., 2000; Jarjour, 2010). The frequency of azoospermia or oligospermia among all FMF patients in our study 
was found to be $1.2 \%$. The total prevalence of male infertility was found to be approximately $5 \%$ (Nagvenkar et al., 2005). The infertility rate in our male FMF patients is lower than that in the normal population. In male FMF patients, progression of the disease may induce testicular impairment, consequently affecting spermatogenesis. However, it is generally assumed that MEFV gene mutations are associated with normal spermatogenesis and therefore, men with FMF are not at increased risk for the genetic factors that predispose to oligospermia and azoospermia, but especially testicular amyloidosis may rarely contribute to infertility in FMF diseases because of the low infertility rate in FMF patients. Our two infertile males with FMF had not received colchicine. The most probable cause of infertility in these patients may be amyloidosis or unknown inflammatory mechanisms in FMF diseases that may cause defects in sperm production.

The most frequent mutations (M680I, M694V, V726A, M694I, and E148Q) in FMF patients are found in more than two-thirds of cases (Brik et al., 1999; Padeh et al., 2003; Olgun et al., 2005). Most of the previous reports covering North African Jewish FMF patients found that M694V is the leading mutation among patients who developed amyloidosis (Dewalle et al., 1998; Pras, 1998; Shohat et al., 1999; Livneh et al., 1999). Ben-Chetrit et al. (1998) reported that nine of 27 patients with FMF had amyloidosis in whom only one was homozygous and three were compound heterozygous for the M694V mutation. The remaining 5 patients with amyloidosis in the same study were found to have different mutations. Two infertile patients with FMF in the present study had different mutations, M680I and M694V homozygosity. Our results show that the heterozygosity or homozygosity of specific mutations are not associated with infertility in this FMF population in the East Anatolia region in Turkey.

Ben-Chetrit et al. (1998) stated that a routine spermiogram is not advised for every male patient with FMF before colchicine treatment. Mijatovic et al. (2003) recommend that a routine spermiogram should be performed in young patients with FMF with renal or other organ amyloidosis. We also believe that a routine spermiogram cannot be recommended for every patient with FMF before colchicine treatment. First, we detected a low infertility rate $(1.2 \%)$ in male patients with newly diagnosed FMF. Second, the effect of the development oligospermia or azoospermia due to colchicine is also rare $(1.3 \%)$ and most patients are diagnosed before puberty. Before starting treatment, we advise untreated patients with renal amyloidosis to have a spermiogram done because amyloidosis may be the cause of azoospermia or oligospermia. Providing genetic counselling in these patients should also be advised to have sperm cryopreservation. The possible adverse effects of colchicine administration on the reproductive potential of treated patients and factors such as amyloidosis on the reproductive potential of untreated patients should be especially monitored with care.

In conclusion, our understanding of the genetic causes of male infertility remains limited. These preliminary data raise several intriguing questions regarding the pathogenesis of infertility in FMF diseases. Do the observed genetic alterations constitute a primary genetic event or not? Does MEFV serve as a modifying gene in spermatogenesis? What is the role of mutations in the MEFV gene in the development of infertility?

The pattern and prevalence of MEFV gene mutations in infertile Turkish men were comparable to those in fertile men. These results suggest that FMF mutations may not be essential for male infertility in the Turkish infertile male population. Our study may also contribute to a better understanding of the factors and mechanisms related to this issue. Further studies should be performed to describe MEFV gene mutation frequencies in the infertile male population and infertility rate in FMF diseases among the Mediterranean and Middle Eastern popula- 
tions, and longitudinal studies involving larger populations are needed to further document the role of pyrin in the control of fertility both under normal conditions and disease states.

\section{ACKNOWLEDGMENTS}

The authors would like to thank all patients and general practitioners of the Firat University Hospital of Elazig for their collaboration in identifying blood donors with no clinical history of infertility. Research supported by Gulpa Medical in Elazig, Turkey.

\section{REFERENCES}

Anonymous (1997). A candidate gene for familial Mediterranean fever. Nat. Genet. 17: 25-31.

Ben-Chetrit E and Levy M (1991). Colchicine prophylaxis in familial Mediterranean fever: reappraisal after 15 years. Semin. Arthritis Rheum. 20: 241-246.

Ben-Chetrit E, Backenroth R, Haimov-Kochman R and Pizov G (1998). Azoospermia in familial Mediterranean fever patients: the role of colchicine and amyloidosis. Ann. Rheum. Dis. 57: 259-260.

Bremner WJ and Paulsen CA (1976). Colchicine and testicular function in man. N. Engl. J. Med. 294: 1384-1385.

Brik R, Shinawi M, Kepten I, Berant M, et al. (1999). Familial Mediterranean fever: clinical and genetic characterization in a mixed pediatric population of Jewish and Arab patients. Pediatrics 103: e70.

Dewalle M, Domingo C, Rozenbaum M, Ben-Chetrit E, et al. (1998). Phenotype-genotype correlation in Jewish patients suffering from familial Mediterranean fever (FMF). Eur. J. Hum. Genet. 6: 95-97.

Ehrenfeld M, Levy M, Margalioth EJ and Eliakim M (1986). The effects of long-term colchicine therapy on male fertility in patients with familial Mediterranean fever. Andrologia 18: 420-426.

Eisenberg S, Aksentijevich I, Deng Z, Kastner DL, et al. (1998). Diagnosis of familial Mediterranean fever by a molecular genetics method. Ann. Intern. Med. 129: 539-542.

Eliakim M, Levy M and Ehrenfeld M (1981). Recurrent Polyserositis (Familial Mediterranean Fever, Periodic disease). Elsevier/North-Holland Biomedical Press, Amsterdam.

Fonnesu C, Cerquaglia C, Giovinale M, Curigliano V, et al. (2009). Familial Mediterranean Fever: a review for clinical management. Joint Bone Spine 76: 227-233.

Gonzalez JL, Gallego E, Castano M and Rueda A (1983). Testicular amyloidosis in hamsters experimentally infected with Leishmania donovani. Br. J. Exp. Pathol. 64: 518-523.

Haimov-Kochman R, Prus D and Ben-Chetrit E (2001). Azoospermia due to testicular amyloidosis in a patient with familial Mediterranean fever. Hum. Reprod. 16: 1218-1220.

Handel MA (1979). Effects of colchicine on spermiogenesis in the mouse. J. Embryol. Exp. Morphol. 51: 73-83.

Jarjour RA (2010). Familial Mediterranean fever in Syrian patients: MEFV gene mutations and genotype-phenotype correlation. Mol. Biol. Rep. 37: 1-5.

Kastrop P, Kimmel I, Bancsi L, Weima S, et al. (1999). The effect of colchicine treatment on spermatozoa: a cytogenetic approach. J. Assist. Reprod. Genet. 16: 504-507.

Levy M and Yaffe C (1978). Testicular function in patients with familial Mediterranean fever on long-term colchicine treatment. Fertil. Steril. 29: 667-668.

Liang JC, Hsu TC and Gay M (1985). Response of murine spermatocytes to the metaphase-arresting effect of several mitotic arrestants. Experientia 41: 1586-1588.

Livneh A, Langevitz P, Shinar Y, Zaks N, et al. (1999). MEFV mutation analysis in patients suffering from amyloidosis of familial Mediterranean fever. Amyloid 6: 1-6.

Medlej-Hashim M, Rawashdeh M, Chouery E, Mansour I, et al. (2000). Genetic screening of fourteen mutations in Jordanian familial Mediterranean fever patients. Hum. Mutat. 15: 384.

Merlin HE (1972). Azoospermia caused by colchicine - a case report. Fertil. Steril. 23: 180-181.

Mijatovic V, Hompes PG and Wouters MG (2003). Familial Mediterranean fever and its implications for fertility and pregnancy. Eur. J. Obstet. Gynecol. Reprod. Biol. 108: 171-176.

Nagvenkar P, Desai K, Hinduja I and Zaveri K (2005). Chromosomal studies in infertile men with oligozoospermia and non-obstructive azoospermia. Indian J. Med. Res. 122: 34-42.

Olgun A, Akman S, Kurt I, Tuzun A, et al. (2005). MEFV mutations in familial Mediterranean fever: association of M694V homozygosity with arthritis. Rheumatol. Int. 25: 255-259. 
Padeh S, Shinar Y, Pras E, Zemer D, et al. (2003). Clinical and diagnostic value of genetic testing in 216 Israeli children with familial Mediterranean fever. J. Rheumatol. 30: 185-190.

Pras M (1998). Familial Mediterranean fever: from the clinical syndrome to the cloning of the pyrin gene. Scand. J. Rheumatol. 27: 92-97.

Rowe PJ, Comhaire FH, Hargreave TB and Mahmoud AM (2000). World Health Organization (WHO) Manual for the Standardized Investigation, Diagnosis and Management of the Infertile Male. 1st edn. Cambridge University Press, Cambridge.

Sarica K, Süzer O, Gürler A, Baltaci S, et al. (1995). Urological evaluation of Behcet patients and the effect of colchicine on fertility. Eur. Urol. 27: 39-42.

Sayarlıoğlu M, Cefle A, Inanç M, Kamali S, et al. (2005). Characteristics of patients with adult-onset familial Mediterranean fever in Turkey: analysis of 401 cases. Int. J. Clin. Pract. 59: 202-205.

Shah K, Sivapalan G, Gibbons N, Tempest H, et al. (2003). The genetic basis of infertility. Reproduction 126: 13-25.

Shinar Y, Livneh A, Langevitz P, Zaks N, et al. (2000). Genotype-phenotype assessment of common genotypes among patients with familial Mediterranean fever. J. Rheumatol. 27: 1703-1707.

Shohat M, Magal N, Shohat T, Chen X, et al. (1999). Phenotype-genotype correlation in familial Mediterranean fever: evidence for an association between Met694Val and amyloidosis. Eur. J. Hum. Genet. 7: 287-292.

Sohar E, Gafni J, Pras M and Heller H (1967). Familial Mediterranean fever. A survey of 470 cases and review of the literature. Am. J. Med. 43: 227-253.

Van Assche E, Bonduelle M, Tournaye H, Joris H, et al. (1996). Cytogenetics of infertile men. Hum. Reprod. 11 (Suppl 4): $1-24$.

Yalcinkaya F, Cakar N, Misirlioğlu M, Tümer N, et al. (2000). Genotype-phenotype correlation in a large group of Turkish patients with familial Mediterranean fever: evidence for mutation-independent amyloidosis. Rheumatology 39: 67-72. 